$\underline{\text { Table of Contents }}$

\title{
Simultaneous Optical Photothermal Infrared (O-PTIR) and Raman Spectroscopy of Submicrometer Atmospheric Particles
}

Nicole E. Olson, ${ }^{1}$ Yao Xiao, ${ }^{1}$ Ziying Lei, ${ }^{2}$ Andrew P. Ault ${ }^{1 *}$

${ }^{1}$ Department of Chemistry, University of Michigan, Ann Arbor, Michigan, 48109

${ }^{2}$ Department of Environmental Health Sciences, University of Michigan, Ann Arbor, Michigan, 48109

*corresponding author's e-mail: aulta@umich.edu

18 Table S1. Number of particles analyzed for each substrate or compound.

19 Table S2. Heat capacity, conductivity, refractive index, and laser power of all compounds used.

20 Figure S1. Spectra of solid ammonium sulfate.

21 Table S3. Spectral modes and images of all substrates tested.

22 Figure S2. Spectra and images of ammonium sulfate particle on gold substrate.

23 Table S4. Spectral assignments and references for all modes identified in Figure 3.

$24 \quad$ Figure S3. Images of all particles analyzed in Figure 3. 
25 Table S1. Number of particles analyzed for each substrate or compound.

\begin{tabular}{|c|c|c|}
\hline Figure & Substrate/Compound & Particles Analyzed \\
\hline \multirow{5}{*}{ Figure 2} & Silicon & 20 \\
\hline & Quartz & 24 \\
\hline & Germanium & 16 \\
\hline & Al Foil & 31 \\
\hline & Ag Foil & 24 \\
\hline \multirow{5}{*}{ Figure 3} & Ammonium Nitrate & 18 \\
\hline & Sodium Acetate & 10 \\
\hline & Sucrose & 12 \\
\hline & $\begin{array}{c}\text { Sodium Dodecyl Sulfate } \\
\text { (SDS) }\end{array}$ & 10 \\
\hline & Ammonium Oxalate & 12 \\
\hline
\end{tabular}

27 
Because O-PTIR signal is dependent on the heat capacity, heat conductivity, and refractive index of the sample, ${ }^{1}$ the authors gathered these values reported in literature for all standards tested. Probe and IR power differed during data collection depending on the sample characteristics and sensitivity to the laser beams. Probe and IR power was varied to maximize signal intensity without oversaturating the detector. The authors list the probe and IR power used for each sample on silicon substrates. The 4 chips for the QCL laser are as follows: $880-1160 \mathrm{~cm}^{-1}, 1160.1-1420 \mathrm{~cm}^{-1}$, $1420.1-1700 \mathrm{~cm}^{-1}$, and $1700.1-1950 \mathrm{~cm}^{-1}$.

The LOD in terms of raw signal is $0.12 \mathrm{~mA}$ from the photodiode. Particles used in the manuscript were generated from $50 \mathrm{mM}$ solutions. To estimate the LOD based on the initial solution concentration, if the volume of a $400 \mathrm{~nm}$ volume equivalent diameter $\left(\mathrm{d}_{\mathrm{ve}}\right)$ particle is $3.35 \times 10^{7}$ $\mathrm{nm}^{3}\left(3.35 \times 10^{-20} \mathrm{~m}^{3}\right)$, then there are $\sim 1.01 \times 10^{6}$ molecules of analyte in that particle before impaction. After impaction the particle spreads to $1 \mu \mathrm{m}$ projected area diameter $\left(\mathrm{d}_{\mathrm{pa}}\right)$ on the substrate. The spot size of the green laser $(\lambda=532 \mathrm{~nm})$ should be similar to the $450 \mathrm{~nm}$ spot size at $\lambda=550 \mathrm{~nm}$ based on manufacturer specs for the Cassegrain objective $(40 \times, 0.78$ N.A. Schwarzschild Objective, IR Reflective, Pike Technologies Inc.). This means would mean we can detect $\sim 4.5 \times 10^{5}$ molecules within the illuminated volume that is determined by the spot size of the green laser.

Table S2. Table of heat capacity, ${ }^{2-9}$ heat conductivity, ${ }^{10,11}$ and refractive index values ${ }^{12-18}$ obtained from literature for all standard compounds tested. On the right, the probe and IR power used to obtain O-PTIR spectra from each standard on silicon substrates is listed.

\begin{tabular}{|c|c|c|c|c|c|}
\hline Compound & $\begin{array}{l}\text { Heat capacity } \\
(\mathbf{J} / \mathbf{g} * \mathbf{C})\end{array}$ & $\begin{array}{c}\text { Heat } \\
\text { conductivity } \\
(\mathbf{W} / \mathbf{m} \times \mathbf{K})\end{array}$ & $\begin{array}{c}\text { Real } \\
\text { refractive } \\
\text { index }\end{array}$ & $\begin{array}{l}\text { Probe power } \\
(\mathrm{mW})\end{array}$ & $\begin{array}{l}\text { IR power } \\
(\mathrm{mW})\end{array}$ \\
\hline Water & 4.18 & 0.60 & 1.33 & $\mathrm{n} / \mathrm{a}$ & $\mathrm{n} / \mathrm{a}$ \\
\hline Ammonium sulfate & 1.42 & $\mathrm{n} / \mathrm{a}$ & 1.85 & 10 & 10 \\
\hline Ammonium nitrate & 1.38 & $\mathrm{n} / \mathrm{a}$ & 1.41 & 10 & 4 \\
\hline Sodium acetate & 0.97 & $\mathrm{n} / \mathrm{a}$ & 1.32 & 4 & 10 \\
\hline Sucrose & 1.04 & $\mathrm{n} / \mathrm{a}$ & 1.36 & 4 & 10 \\
\hline $\begin{array}{l}\text { Sodium dodecyl } \\
\text { sulfate }\end{array}$ & 1.14 & $\mathrm{n} / \mathrm{a}$ & 1.33 & 4 & 10 \\
\hline $\begin{array}{l}\text { Ammonium } \\
\text { oxalate }\end{array}$ & 1.05 & $\mathrm{n} / \mathrm{a}$ & 1.34 & 10 & 5 \\
\hline
\end{tabular}



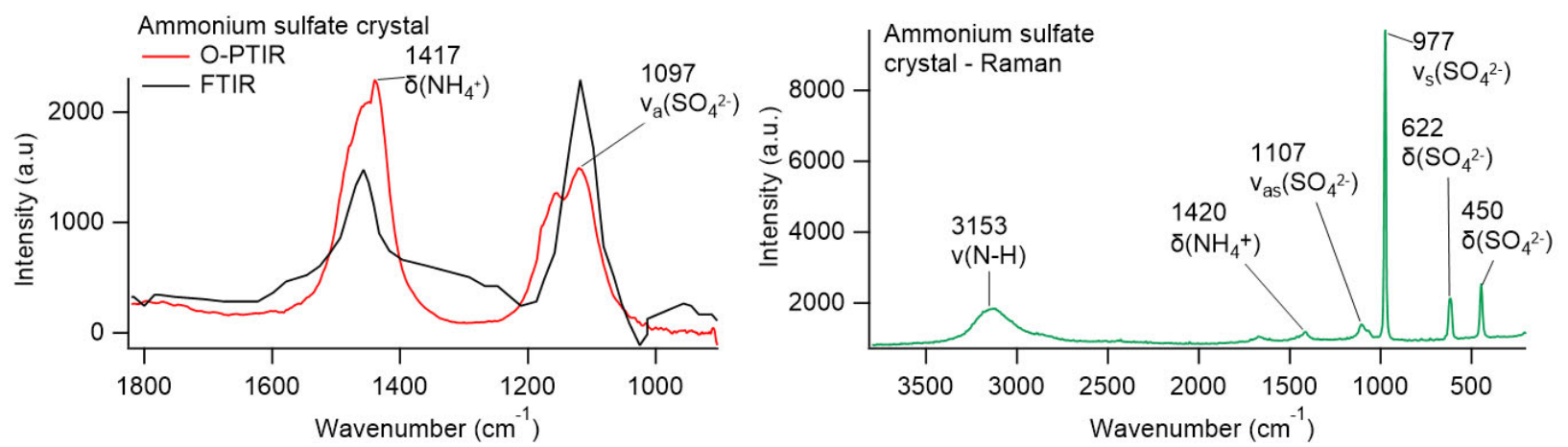

Figure S1. IR (left, red trace) and Raman (right, green trace) spectra of ammonium sulfate crystals, illustrating the crystalline modes observed by Raman. ${ }^{19}$ O-PTIR and FTIR spectra of ammonium sulfate aligns well, similar to previous comparison of photothermal IR and FTIR. ${ }^{20}$

Table S3. Table listing peaks identified by IR and Raman for each substrate tested. Optical

60 images of blank substrates (with no particles) are included on the right.

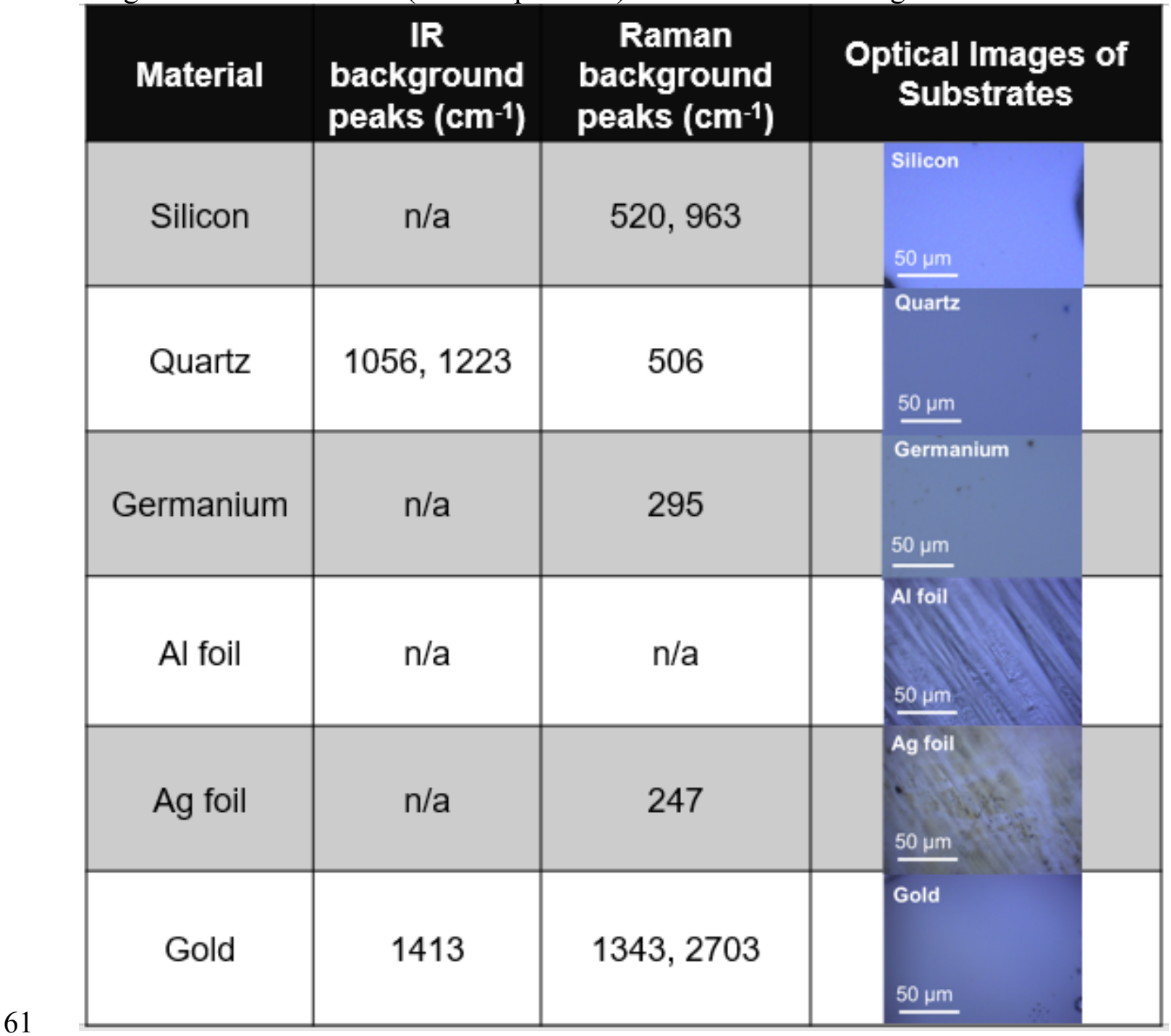



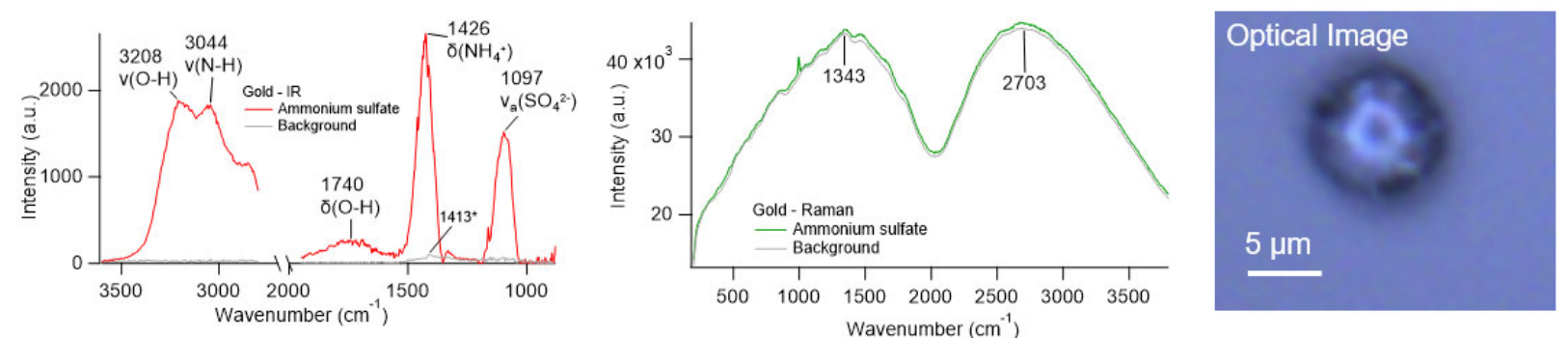

Figure S2. IR (left, red trace) and Raman (right, green trace) spectra obtained from an ammonium sulfate particle on a gold substrate. Raman spectra were dominated from fluorescence due to the substrate. An optical image of the particle is included on the right.

Table S4. Table listing all vibrational modes experimentally determined in IR and Raman spectra. Literature is referenced to corroborate the spectral assignments. ${ }^{19-42}$

\begin{tabular}{|c|c|c|c|c|}
\hline Compound & Experimental IR modes & IR References & Experimental Raman modes & Raman References \\
\hline Ammonium sulfate & $\begin{array}{c}v_{\mathrm{as}}\left(\mathrm{SO}_{4}{ }^{2-}\right) \text { at } 1100 \mathrm{~cm}^{-1} \\
\delta\left(\mathrm{NH}_{4}^{+}\right) \text {at } 1422 \\
v(\mathrm{~N}-\mathrm{H}) \text { at } \sim 3040 \mathrm{~cm}^{-1}\end{array}$ & $\begin{array}{l}\text { Onasch et al. } 1999 \\
\text { Bondy et al. } 2017 \\
\text { Or et al. } 2018\end{array}$ & $\begin{array}{c}v_{\mathrm{s}}\left(\mathrm{SO}_{4}{ }^{2-}\right) \text { at } 977 \mathrm{~cm}^{-1} \\
\delta\left(\mathrm{SO}_{4}{ }^{-2}\right) \text { at } 634 \text { and } 471 \mathrm{~cm}^{-1} \\
v(\mathrm{~N}-\mathrm{H}) \text { at } \sim 3168 \mathrm{~cm}^{-1}\end{array}$ & $\begin{array}{c}\text { Venkateswarlu et al. } 1975 \\
\text { Jentzsch et al. } 2013 \\
\text { Rindelaub et al. } 2016 \\
\text { Kirpes et al. } 2018\end{array}$ \\
\hline Ammonium nitrate & $\begin{array}{l}v_{a}\left(\mathrm{NO}_{3}^{-}\right) \text {at } 1371 \mathrm{~cm}^{-1} \\
\delta\left(\mathrm{NH}_{4}^{+}\right) \text {at } 1411 \mathrm{~cm}^{-1} \\
v(\mathrm{~N}-\mathrm{H}) \text { at } 3081 \mathrm{~cm}^{-1}\end{array}$ & $\begin{array}{c}\text { Theoret et al. } 1964 \\
\text { Bondy et al. } 2017 \\
\text { Or et al. } 2018\end{array}$ & $\begin{array}{l}v\left(\mathrm{NO}_{3}^{-}\right) \text {at } 1044 \mathrm{~cm}^{-1} \\
v(\mathrm{~N}-\mathrm{H}) \text { at } 3135 \mathrm{~cm}^{-1}\end{array}$ & $\begin{array}{l}\text { Rousseau et al. } 1968 \\
\text { Jentzsch et al. } 2013 \\
\text { Craig et al. } 2015 \\
\text { Craig et al. } 2017\end{array}$ \\
\hline Sodium acetate & $\begin{array}{c}v\left(\mathrm{CH}_{3}\right) \text { at } 1361 \mathrm{~cm}^{-1} \\
v(\mathrm{C}-\mathrm{O}) \text { at } 1411 \mathrm{~cm}^{-1} \\
v_{\mathrm{a}}(\mathrm{C}=\mathrm{O}) \text { at } 1577 \mathrm{~cm}^{-1} \\
v(\mathrm{C}-\mathrm{H}) \text { at } 2936 \text { and } 3004 \mathrm{~cm}^{-1}\end{array}$ & $\begin{array}{c}\text { Jones et al. } 1955 \\
\text { Jones et al. } 1954 \\
\text { Kakihana et al. } 1982\end{array}$ & $\begin{array}{c}v\left(\mathrm{CH}_{3}\right) \text { at } 1360 \mathrm{~cm}^{-1} \\
v(\mathrm{C}-\mathrm{H}) \text { at } 2717,2829,2935 \mathrm{~cm}^{-1}\end{array}$ & $\begin{array}{l}\text { Frost et al. } 2000 \\
\text { Wang et al. } 2005\end{array}$ \\
\hline Sucrose & $\begin{array}{c}v(\mathrm{C}-\mathrm{C}) \text { at } 935 \mathrm{~cm}^{-1} \\
v(\mathrm{C}-\mathrm{O}) \text { at } 1054,1128 \mathrm{~cm}^{-1} \\
\delta(\mathrm{C}-\mathrm{O}-\mathrm{H}) \text { at } 1408 \mathrm{~cm}^{-1} \\
v\left(\mathrm{CH}_{2}\right) \text { at } 2916 \mathrm{~cm}^{-1} \\
v(\mathrm{O}-\mathrm{H}) \text { at } 3356 \mathrm{~cm}^{-1}\end{array}$ & $\begin{array}{l}\text { Brizuela et al. } 2012 \\
\text { Or et al. } 2018\end{array}$ & $\begin{array}{l}v\left(\mathrm{CH}_{2}\right) \text { at } 836 \mathrm{~cm}^{-1} \\
v(\mathrm{C}-\mathrm{O}) \text { at } 1132 \mathrm{~cm}^{-1} \\
v\left(\mathrm{CH}_{2}\right) \text { at } 1339 \mathrm{~cm}^{-1} \\
\delta\left(\mathrm{CH}_{2}\right) \text { at } 1462 \mathrm{~cm}^{-1} \\
v(\mathrm{C}-\mathrm{H}) \text { at } 2910 \mathrm{~cm}^{-1} \\
v(\mathrm{O}-\mathrm{H}) \text { at } 3345 \mathrm{~cm}^{-1}\end{array}$ & $\begin{array}{l}\text { Mathlouthi et al. } 1980 \\
\text { Brizuela et al. } 2012\end{array}$ \\
\hline Sodium dodecyl sulfate & $\begin{array}{c}v_{\text {as }}\left(\mathrm{ROSO}_{3}^{-}\right) \text {at } 1221,1249 \mathrm{~cm}^{-1} \\
\delta\left(\mathrm{CH}_{2}\right) \text { at } 1469 \mathrm{~cm}^{-1} \\
v(\mathrm{CH}) \text { at } 2885,2921,2955 \mathrm{~cm}^{-1} \\
v(\mathrm{O}-\mathrm{H}) \text { at } 3471 \mathrm{~cm}^{-1}\end{array}$ & $\begin{array}{l}\text { Sperline et al. } 1992 \\
\text { Gao et al. } 2010\end{array}$ & $\begin{array}{c}v\left(\mathrm{ROSO}_{3}\right) \text { at } 1086 \mathrm{~cm}^{-1} \\
v(\mathrm{C}-\mathrm{C}) \text { at } 1270 \mathrm{~cm}^{-1} \\
\delta(\mathrm{C}-\mathrm{C}) \text { at } 1441 \mathrm{~cm}^{-1} \\
v(\mathrm{C}-\mathrm{H}) \text { at } 2727,2846,2878 \mathrm{~cm}^{-1}\end{array}$ & $\begin{array}{l}\text { Picquart et al. } 1986 \\
\text { Ault et al. } 2013\end{array}$ \\
\hline Ammonium oxalate & $\begin{array}{c}v(\mathrm{C}-\mathrm{O}) \text { at } 1302,1439 \mathrm{~cm}^{-1} \\
v(\mathrm{C}=\mathrm{O}) \text { at } 1719 \mathrm{~cm}^{-1} \\
v(\mathrm{~N}-\mathrm{H}) \text { at } 3000 \mathrm{~cm}^{-1} \\
v(\mathrm{O}-\mathrm{H}) \text { at } 3196 \mathrm{~cm}^{-1}\end{array}$ & Frost et al. 2003 & $\begin{array}{c}v(\mathrm{C}-\mathrm{C}) \text { at } 875 \mathrm{~cm}^{-1} \\
v(\mathrm{C}-\mathrm{O}) \text { at } 1452,1476 \mathrm{~cm}^{-1} \\
v(\mathrm{~N}-\mathrm{H}) \text { at } 3029 \mathrm{~cm}^{-1}\end{array}$ & $\begin{array}{l}\text { Hibben et al. } 1935 \\
\text { Frost et al. } 2003\end{array}$ \\
\hline
\end{tabular}


Ammonium Nitrate

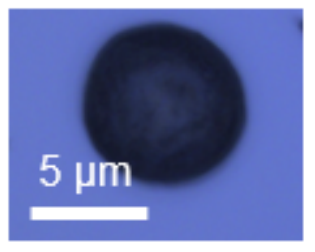

\section{Sodium} Acetate

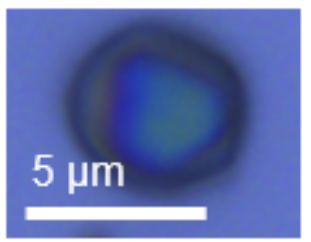

Sucrose

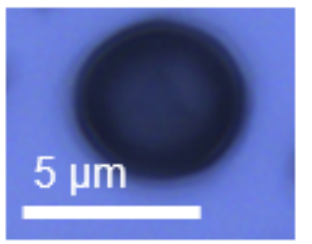

\section{SDS}

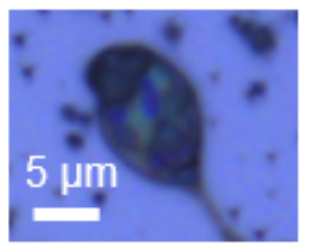

Ammonium Oxalate

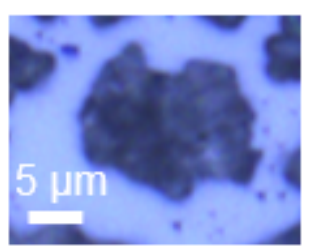

78 Figure S3. Optical images of each particle analyzed in Figure 3. All particles had an aerodynamic 79 diameter $<400 \mathrm{~nm}$ before spreading onto the silicon substrate. 
1. Zhang, D., Li, C., Zhang, C., Slipchenko, M. N., Eakins, G., Cheng, J. X., Depth-resolved midinfrared photothermal imaging of living cells and organisms with submicrometer spatial resolution. Sci. Adv. 2016, 2, (9), e1600521.

2. Latimer, W. M., Schutz, P. W., Hicks, J. F. G., The heat capacity and entropy of calcium oxalate from 19 to $300^{*}$ absolute. The entropy and free energy of oxalate ion. J. Am. Chem. Soc. 1993, 55, (3), 971-975.

3. Angell, C. A., Sichina, J. W., Oguni, M., Heat capacity of water at extremes of supercooling and superheating. J. Phys. Chem. 1982, 86, (6), 998-1002.

4. Zhou, S.-Q.; Ni, R., Measurement of the specific heat capacity of water-based Al2O3 nanofluid. Appl. Phys. Lett. 2008, 92, (9), 093123.

5. Clegg, S. L., Ho, S. S., Chan, C. K., Brimblecombe, P., Thermodynamic properties of aqueous (NH4)2SO4 to high supersaturation as a function of temperature. J. Chem. Eng. Data 1995, 40, 1079-1090.

6. Stephenson, C. C., Bentz, D. R., Stevenson, D. A., The heat capacity of ammonium nitrate from 15 to 315 K. J. Am. Chem. Soc. 1955, 77, (8), 2161-2164.

7. Magoń, A.; Wurm, A.; Schick, C.; Pangloli, P.; Zivanovic, S.; Skotnicki, M.; Pyda, M., Heat capacity and transition behavior of sucrose by standard, fast scanning and temperaturemodulated calorimetry. Thermochim. Acta. 2014, 589, 183-196.

8. Araki, N., Futamura, M., Makino, A., Shibata, H., Measurements of thermophysical properties of sodium acetate hydrate. Int. J. Thermophys. 1995, 16, (6), 1455-1466.

9. Bakshi, M. S., Crisantino, R., De Lisi, R., Milioto, S., Volume and heat capacity of sodium dodecyl sulfate-dodecyldimethylamine oxide mixed micelles. J. Phys. Chem. 1993, 97, 6914-6919.

10. Sengers, J. V.; Watson, J. T. R.; Basu, R. S.; Kamgar-Parsi, B.; Hendricks, R. C., Representative equations for the thermal conductivity of water substance. J. Phys. Chem. Ref. Data 1984, 13, (3), 893-933.

11. Zhang, M.; Che, Z.; Chen, J.; Zhao, H.; Yang, L.; Zhong, Z.; Lu, J., Experimental determination of thermal conductivity of water-agar gel at different concentrations and temperatures. J. Chem. Eng. Data 2011, 56, (4), 859-864.

12. Hale, G. M., Querry, M. R., Optical constants of water in the 200-nm to 200-um wavelength region. Appl. Opt. 1973, 12, (3), 555-563.

13. Earle, M. E., Pancescu, R. G., Cosic, B., Zasetsky, A. Y., Sloan, J. J., Temperature-dependent complex indices of refraction for crystalline (NH4)2SO4. J. Phys. Chem. A 2006, 110, $13022-13028$.

14. Jarzembski, M. A., Norman, M. L., Fuller, K. A., Srivastava, V., Cutten, D. R., Complex refractive index of ammonium nitrate in the $2-20 \mathrm{~nm}$ spectral range. Appl. Opt. 2003, 42, (6), 922-930.

15. Deosarkar, S. D.; Mendkudle, M. S., Physicochemical properties and ion-solvent interactions in aqueous sodium, ammonium, and lead acetate solution. Russ. J. Phys. Chem. A 2014, $88,(9), 1527-1532$.

16. Charles, D. F., Refractive indices of sucrose-water solutions in the range from 24 ro $53 \%$ sucrose. Anal. Chem. 1965, 37, (3), 405-406.

17. Singh, R.; Chauhan, S.; Sharma, K., Surface tension, viscosity, and refractive index of sodium dodecyl sulfate (SDS) in aqueous solution containing polyethylene glycol (PEG), 
polyvinyl pyrrolidone (PVP), and their blends. J. Chem. Eng. Data 2017, 62, (7), 19551964.

18. Frej, H., Jakubczyk, M., Density, surface tension, and refractive index of aqueous ammonium oxalate solutions from 293 to 333 K. J. Chem. Eng. Data 1998, 43, 158-161.

19. Venkateswarlu, P., Bist, H. D., Jain, Y. S., Laser excited Raman spectrum of ammonium sulfate single crystal. J. Raman Spectrosc. 1975, 3, 143-151.

20. Bondy, A. L.; Kirpes, R. M.; Merzel, R. L.; Pratt, K. A.; Banaszak Holl, M. M.; Ault, A. P., Atomic force microscopy-infrared spectroscopy of individual atmospheric aerosol particles: subdiffraction limit vibrational spectroscopy and morphological analysis. Anal. Chem. 2017, 89, 8594-8598.

21. Or, V. W., Estillore, A. D., Tivanski, A. V., Grassian, V. H., Lab on a tip: atomic force microscopy - photothermal infrared spectroscopy of atmospherically relevant organic/inorganic aerosol particles in the nanometer to micrometer size range. Analyst 2018, 143, 2765-2774.

22. Onasch, T. B.; Siefert, R. L.; Brooks, S. D.; Prenni, A. J.; Murray, B.; Wilson, M. A.; Tolbert, M. A., Infrared spectroscopic study of the deliquescence and efflorescence of ammonium sulfate aerosol as a function of temperature. J Geophys Res Atmos 1999, 104, 21317-21326.

23. Craig, R. L.; Bondy, A. L.; Ault, A. P., Computer-controlled Raman microspectroscopy (CCRaman): A method for the rapid characterization of individual atmospheric aerosol particles. Aerosol. Sci. Technol. 2017, 51, 1099-1112.

24. Craig, R. L.; Nandy, L.; Axson, J. L.; Dutcher, C. S.; Ault, A. P., Spectroscopic determination of aerosol $\mathrm{pH}$ from acid-base equilibria in inorganic, organic, and mixed systems. J. Phys. Chem. A 2017, 121, (30), 5690-5699.

25. Kirpes, R. M.; Bondy, A. L.; Bonanno, D.; Moffet, R. C.; Wang, B.; Laskin, A.; Ault, A. P.; Pratt, K. A., Secondary sulfate is internally mixed with sea spray aerosol and organic aerosol in the winter Arctic. Atmos. Chem. Phys. 2018, 18, (6), 3937-3949.

26. Rindelaub, J. D.; Craig, R. L.; Nandy, L.; Bondy, A. L.; Dutcher, C. S.; Shepson, P. B.; Ault, A. P., Direct measurement of $\mathrm{pH}$ in individual particles via Raman microspectroscopy and variation in acidity with relative humidity. J. Phys. Chem. A 2016, 120, (6), 911-917.

27. Theoret, A., Sandorfy, C., Infrared spectra and crystalline phase transitions of ammonium nitrate. Can. J. Chem. 1964, 42, 57-62.

28. Craig, R. L.; Bondy, A. L.; Ault, A. P., Surface enhanced Raman spectroscopy enables observations of previously undetectable secondary organic aerosol components at the individual particle level. Anal. Chem. 2015, 87, (15), 7510-7514.

29. Jones, L. H., Infrared spectra and structure of the crystalline sodium acetate complexes of $\mathrm{U}(\mathrm{VI}), \mathrm{Np}(\mathrm{VI}), \mathrm{Pu}(\mathrm{VI})$, and Am(VI). A comparison of metal-oxygen bond distance and bond force constant in this series. J. Chem. Phys. 1955, 23, (11), 2105-2107.

30. Jones, L. H.; McLaren, E., Infrared spectra of CH3COONa and CD3COONa and assignments of vibrational frequencies. J. Chem. Phys. 1954, 22, (11), 1796-1800.

31. Kakihana, M., Kotaka, M., Okamoto, M., Infrared spectra of 13C-substituted species of solid sodium acetate at 80 K. J. Phys. Chem. 1982, 86, (22), 4385-4387.

32. Wang, L. Y., Zhang, Y. H., Zhao, L. J., Raman spectroscopic studies on single supersaturated droplets of sodium and magnesium acetate. J. Phys. Chem. A 2005, 109, 609-614.

33. Frost, R. L., Kloprogge, J. T., Raman spectroscopy of the acetates of sodium, potassium, and magnesium at liquid nitrogen temperature J. Mol. Struct. 2000, 526, 131-141. 
34. Brizuela, A. B.; Bichara, L. C.; Romano, E.; Yurquina, A.; Locatelli, S.; Brandan, S. A., A complete characterization of the vibrational spectra of sucrose. Carbohydr Res 2012, 361, 212-218.

35. Gao, X.; Chorover, J., Adsorption of sodium dodecyl sulfate (SDS) at ZnSe and alpha-Fe2O3 surfaces: combining infrared spectroscopy and batch uptake studies. J Colloid Interface Sci 2010, 348, (1), 167-176.

36. Ault, A. P.; Zhao, D.; Ebben, C. J.; Tauber, M. J.; Geiger, F. M.; Prather, K. A.; Grassian, V. H., Raman microspectroscopy and vibrational sum frequency generation spectroscopy as probes of the bulk and surface compositions of size-resolved sea spray aerosol particles. Phys. Chem. Chem. Phys. 2013, 15, (17), 6206-6214.

37. Frost, R. L., Jing, Y., Ding, Z., Raman and FTIR spectroscopy of natural oxalates: Implications for life on Mars. Chinese Sci. Bull. 2003, 48, (17), 1844-1852.

38. Rousseau, D. L., Miller, R. E., Leroi, G. E., Raman spectra of crystalline sodium nitrate. J. Chem. Phys. 1968, 48, (8), 3409.

39. Jentzsch, P. V.; Kampe, B.; Ciobota, V.; Rosch, P.; Popp, J., Inorganic salts in atmospheric particulate matter: Raman spectroscopy as an analytical tool. Spectrochim. Acta A Molec. Biomolec. Spectrosc. 2013, 115, 697-708.

40. Picquart, M.; Laborde, M., Raman Scattering in Aqueous Solutions of Sodium Dodecyl Sulfate. In Surfactants in Solution: Volume 4, Mittal, K. L.; Bothorel, P., Eds. Springer US: Boston, MA, 1986; pp 189-201.

41. Hibben, J. H., The Raman spectra of oxalic acid. J. Chem. Phys. 1935, 3, 675-679.

42. Mathlouthi, M., Luu, D. V., Laser-Raman spectra of D-glucose and sucrose in aqueous solution. Carbohydr Res 1980, 81, (2), 203-212. 